\title{
Criminologie
}

\section{Quelques notes sur la réforme de la détermination de la peine au Canada (1991)}

\section{Jean-Paul Brodeur et Marion Vacheret}

Volume 44, numéro 1, printemps 2011

Jean-Paul Brodeur, d'hier à aujourd'hui

URI : https://id.erudit.org/iderudit/1001604ar

DOI : https://doi.org/10.7202/1001604ar

Aller au sommaire du numéro

\section{Éditeur(s)}

Les Presses de l’Université de Montréal

ISSN

0316-0041 (imprimé)

1492-1367 (numérique)

Découvrir la revue

Citer cet article

Brodeur, J.-P. \& Vacheret, M. (2011). Quelques notes sur la réforme de la détermination de la peine au Canada (1991). Criminologie, 44(1), 97-118. https://doi.org/10.7202/1001604ar

\section{Résumé de l'article}

Une recension des développements récents au sujet de la détermination de la peine permet de constater que depuis que la Commission canadienne sur la détermination de la peine a déposé son rapport de 1987, aucune de ses recommandations n'a été mise en oeuvre. Cela constitue une prolongation du statu quo. Nous proposons des éléments d'explication en réponse à pourquoi il n'y a eu aucun suivi des propositions de la Commission. Deuxièmement, nous présentons une analyse critique du rapport de la consultation sur la détermination de la peine et la libération conditionnelle présenté en 1990 par le ministère fédéral de la Justice. Nous soutenons que les déclarations d'intention et les principes de détermination de la peine proposés devraient établir des priorités parmi les divers objectifs qui y sont énumérés. Nous soulignons également qu'il y a un déséquilibre entre les composantes de la détermination de la peine et celles de la libération conditionnelle de la commission permanente tel que proposé par le ministère de la Justice. La composante de la détermination de la peine doit constituer une politique de détermination de la peine alors que la composante de libération conditionnelle doit être un avis à la Commission nationale des libérations conditionnelles. 


\section{Quelques notes sur la réforme de la détermination de la peine au Canada}

Jean-Paul Brodeur $\left(1991^{\star}\right)$

\section{Note introductive}

En 1991, quand Jean-Paul Brodeur écrit cet article, la Commission canadienne sur la détermination de la peine a publié depuis plus de quatre ans son imposant projet de réforme sans quaucune modification n'ait été adoptée en la matière. La tradition de palabres infinies qui semble être une des spécialités du gouvernement canadien de l'époque, les conflits politiques, l'activisme de groupes de pression et surtout les divisions provinciales et fédérales expliquent pour partie cette inertie qui durera longtemps. Visionnaire désabusé, Jean-Paul Brodeur critique l'absence d'action du gouvernement tout en en s'en félicitant. Que n'avait-il raison! Les modifications - non pensées, non justifiées, non fondées sauf à défendre un populisme de bas étage - aujourd'hui affluent.

Les premiers changements, prévoyant à la fois un durcissement pénal et la création du sursis comme «mesure alternative» et amorcés en 1996, seront qualifiés de schizophrènes (le mot est d'Hélène Dumont), en raison de leur ambiguiité. Depuis, l'inflation législative se concentre sur la démultiplication de mesures de sûreté - peines minimales obligatoires, ordonnances de surveillance de longue durée, renforcement des lois sur les délinquants dits «dangereux» - et sur les restrictions dans l'accès à des mesures non carcérales ou de libérations anticipées. Près de 20 ans après les réflexions de Jean-Paul Brodeur, force est de constater que

${ }^{\star}$ Criminologie, XXIV (2), 81-98.

Criminologie, vol. $44, \mathrm{n}^{\circ} 1$ (2011) 
l'inertie en matière de sentencing, inertie longtemps critiquée et dénoncée, a été remplacée par un activisme répressif forcené.

Aujourd'hui, à défaut d'être mesurées, les recommandations des groupes d'études nommés par le gouvernement, tout comme les législations déposées par le parti au pouvoir, sont directes: sus aux délinquants et haro sur les mesures trop douces! Les préoccupations de Jean-Paul Brodeur face à l'inconséquence des différents groupes d'étude comme son souhait du maintien d'un statu quo, bénéfique selon lui pour la clarté des décisions, n’ont, hélas, apparemment plus lieu d'être.

Marion Vacheret ${ }^{\star \star}$

RÉSUMÉ - Une recension des développements récents au sujet de la détermination de la peine permet de constater que depuis que la Commission canadienne sur la détermination de la peine a déposé son rapport de 1987, aucune de ses recommandations n'a été mise en œuvre. Cela constitue une prolongation du statu quo. Nous proposons des éléments d'explication en réponse à pourquoi il n'y a eu aucun suivi des propositions de la Commission. Deuxièmement, nous présentons une analyse critique du rapport de la consultation sur la détermination de la peine et la libération conditionnelle présenté en 1990 par le ministère fédéral de la Justice. Nous soutenons que les déclarations d'intention et les principes de détermination de la peine proposés devraient établir des priorités parmi les divers objectifs qui y sont énumérés. Nous soulignons également qu'il y a un déséquilibre entre les composantes de la détermination de la peine et celles de la libération conditionnelle de la commission permanente tel que proposé par le ministère de la Justice. La composante de la détermination de la peine doit constituer une politique de détermination de la peine alors que la composante de libération conditionnelle doit être un avis à la Commission nationale des libérations conditionnelles.

MOTS-CLÉS - Commission canadienne sur la détermination de la peine, politique de détermination de la peine, libération conditionnelle, Commission nationale des libérations conditionnelles, ministère de la Justice du Canada.

\section{Introduction}

Pour des raisons de commodité, nous désignerons le processus de détermination de la peine par l'appellation de sentencing. Comme nous venons tout juste de l'indiquer, le sentencing est un processus. En luimême, il réside dans l'imposition d'une sentence par les tribunaux. L'imposition de la sentence est néanmoins précédée par une étape où

\footnotetext{
${ }^{\star \star}$ Professeure, École de criminologie, Université de Montréal

Chercheure

Centre international de criminologie comparée (CICC)

marion.vacheret@umontreal.ca
} 
le ministère public détermine la nature des accusations qui sont portées ainsi que leur nombre. On nomme fréquemment cette étape négociation du plaidoyer (cette désignation n'est pas la seule possible: on peut aussi se référer à ce processus sous l'appellation de communication de la preuve). Le sentencing proprement dit est également suivi d'une étape d'application de la sentence déterminée par le juge. Ce sont les services correctionnels qui sont responsables de l'application des peines. Le sentencing est donc un processus qui est précédé d'une étape et qui est suivi d'une autre.

Même lorsque l'on considère le sentencing dans toutes ses étapes, on ne saurait nier qu'il a fait l'objet d'un travail considérable effectué par diverses commissions d'enquête et par d'autres organismes. Énumérons rapidement les principaux travaux. La Commission canadienne sur la détermination de la peine (CCDP) a remis son rapport en février 1987. Ce rapport contient des recommandations sur tous les aspects du sentencing. Le rapport de la CCDP a été suivi par les travaux du Comité permanent de la justice et du Solliciteur général sur la détermination de la peine, la mise en liberté sous condition et d'autres aspects du système correctionnel (CJSG). Ce comité a remis son rapport en août 1988. Ce rapport portait très exactement sur les mêmes questions que celles débattues dans le rapport de la CCDP.

En outre, la Commission de réforme du droit pénal du Canada (CRDP) a publié en 1989 un important document qui s'intitule Les discussions et ententes sur le plaidoyer. Comme son titre l'indique, ce document de travail porte sur l'étape préliminaire au sentencing. En 1987, la CRDP avait également publié un rapport intitulé Pour une nouvelle codification du droit pénal. Ce rapport n'est rien de moins qu'une recodification de la Partie générale et de la Partie spéciale du Code criminel du Canada.

On peut affirmer de façon générale que ces travaux n'ont été suivis d'aucune application, si ce n'est quelques réformes administratives qui ont été effectuées dans le domaine correctionnel et dans celui des libérations conditionnelles. En effet, le ministère de la Justice du Canada a publié en 1990 deux documents de consultation qui portent un même titre (Vers une réforme). Le premier de ces documents porte sur le sentencing (la détermination de la peine). Le second porte quant à lui sur les affaires correctionnelles et la mise en liberté sous condition. Il importe de souligner que ces deux documents ne contiennent pas des propositions finales. Ils sont soumis aux divers intervenants qui œuvrent tant dans le domaine public qu'à titre privé pour recueillir leur avis sur des 
propositions ouvertes. Ces propositions sont en effet exprimées sous forme d'alternatives. On doit donc conclure qu'en dépit de tous les travaux conduits par les diverses commissions et comités que nous avons énumérés plus haut, le gouvernement fédéral en est toujours à une étape de consultation. La dernière consultation entreprise en 1990 est loin d'être terminée. On peut prévoir qu'elle donnera lieu à la rédaction d'un rapport qui discutera à nouveau des questions abordées par la CCDP en 1987, par le CJSG en 1988 et par la CRDP depuis 1970. On ne saurait donc espérer que des réformes soient effectuées dans un proche avenir.

Les remarques qui précèdent valent surtout au niveau des réformes du processus de détermination de la peine qui auraient pu être effectuées par le gouvernement fédéral. Comme nous le verrons, les commissions canadiennes des libérations conditionnelles, ainsi que les services correctionnels fédéraux ont déjà procédé à l'implantation d'un certain nombre de mesures réformatrices. En outre, il n'est pas douteux que des réformes et des expériences aient été effectuées par les provinces. Par exemple, la Colombie-Britannique a déjà réalisé des projets pilotes dans le domaine de l'assignation à domicile sous surveillance électronique. Ces projets ont fait l'objet d'évaluations qui ont révélé qu'ils entraînaient des frais en personnel considérables. Nous n'avons pu effectuer une recension complète des initiatives pénales provinciales dans le cadre de la préparation de ce texte, qui n'est qu'une simple note.

Dans les pages qui vont suivre, nous allons d'abord nous interroger sur les raisons de la présente inertie du gouvernement fédéral. En second lieu, nous présenterons quelques commentaires sur les deux documents de consultation distribués en 1990 par le ministère de la Justice et celui du Solliciteur général du Canada.

\section{Un certain piétinement}

Avant d'avancer un certain nombre de raisons pour expliquer que le processus de réforme semble s'être embourbé, il importe de rappeler, par souci d'équité, que les réformes d'importance prennent un temps considérable. Ainsi, la réforme de la législation sur les jeunes contrevenants est un processus qui s'est étendu sur quelque vingt-trois ans (de 1961 à 1984). Lors de nos conversations avec des fonctionnaires du gouvernement fédéral, nous avons pu constater que la réforme du sentencing était souvent comparée à celle de la justice des mineurs. On s'attendait donc à ce que le processus s'étale sur plusieurs années. Ceci 
étant dit, nous pouvons tout de même faire quelques considérations sur les facteurs qui ont constitué des obstacles aux réformes. Qu'il soit toutefois bien entendu que nos remarques ne sont pas dictées par l'impatience, ni même par le dépit, car nous sommes bien conscient que les réformes proposées par la CCDP impliquent des changements majeurs dans tout le système pénal.

\subsection{La question politique}

Il importe d'abord de discuter une question rarement abordée dans le domaine de la réforme du sentencing, sauf aux États-Unis. La justice pénale a fait l'objet d'une intense politisation, depuis le début des années 1980. À cet égard, nous remarquerons que les commissaires qui ont siégé à la CCDP ont tous été nommés en mai 1984 par un gouvernement, lequel était formé à l'époque par le Parti libéral. Ce gouvernement était alors en fin de mandat et s'apprêtait à affronter une élection. Le Parti libéral perdit cette élection aux mains du Parti conservateur et de son candidat, monsieur Brian Mulroney, qui devint donc Premier ministre du Canada. Il est, croyons-nous, inutile de se demander quelle était véritablement l'appartenance politique des commissaires de la CCDP. Certains de ces commissaires étaient identifiés comme des libéraux, d'autres pas du tout. Quoi qu'il en soit, il est difficile d'échapper à la conclusion que le nouveau gouvernement ne pouvait accueillir dans l'enthousiasme le rapport d'une commission d'enquête qui avait été créée par le gouvernement libéral précédent. En effet, les positions du Parti libéral et du Parti conservateur en matière de justice pénale diffèrent notablement. Il faut en effet se souvenir qu'au moment même où la CCDP préparait son rapport sur la désescalade des sanctions, le gouvernement conservateur donnait suite à sa promesse de tenir un débat sur le rétablissement de la peine de mort au Canada. Le rapport de la CCDP fut publié dans les semaines qui précédèrent le vote sur le rétablissement de la peine de mort au Canada. Sa publication passa relativement inaperçue dans les médias à cause du débat passionné que suscitait le rétablissement de la peine de mort. Or, on sait que la pression exercée par les médias sur le gouvernement est l'un des atouts les plus décisifs dont puisse jouir une commission d'enquête. La CCDP ne put véritablement disposer de cet atout.

Lors de la conférence de presse qui fut tenue par les membres de la CCDP au moment de la publication de son rapport, l'ancien Solliciteur 
général du Canada, monsieur Bob Kaplan, reprocha publiquement à cette commission de provoquer un durcissement de l'opinion publique en faveur du rétablissement de la peine de mort. La CCDP proposait en effet que la peine obligatoire de 25 ans d'incarcération pour meurtre au premier degré soit diminuée et qu'un détenu puisse bénéficier d'une libération conditionnelle après 15 ans d'incarcération. Suivant le raisonnement de monsieur Kaplan, cette recommandation équivalait à jeter de l'huile sur le feu.

Il est difficile de produire des conclusions définitives sur l'influence des facteurs de nature politique sur le manque de volonté du gouvernement de donner une suite aux recommandations de la CCDP. On peut néanmoins voir dans le mandat qui fut donné au Comité permanent de la justice et du Solliciteur général sur la détermination de la peine, la mise en liberté sous condition et d'autres aspects du système correctionnel (CJSG), présidé par monsieur David Daubney, un député conservateur, un indice du fait que le gouvernement voulait recevoir des propositions d'un comité en majorité composé de membres du Parti conservateur. En effet, il est sinon difficile de s'expliquer pourquoi le gouvernement conservateur allait donner au CJSG le mandat de se pencher exactement sur les mêmes questions qui avaient été abordées en détail par la CCDP. Pourquoi donc avoir dépensé quelques millions de dollars pour défrayer les travaux d'une commission d'enquête et décider dans les semaines suivant le dépôt du rapport de cette commission que le travail devait être repris?

Monsieur Daubney déposa son rapport en août 1988. Ce rapport fut généralement bien accueilli et sa prise de position en faveur des libérations conditionnelles fut retenue par le gouvernement fédéral. Il importe de souligner que monsieur Daubney ne fut pas réélu aux élections fédérales subséquentes. Il fut donc mis dans l'impossibilité de militer à l'intérieur du gouvernement pour l'application des recommandations les plus novatrices de son rapport. En effet, le gouvernement ne retint du rapport Daubney que les recommandations qui consolidaient le statu quo.

\subsection{Les groupes de pression}

La CCDP recommanda l'abolition des libérations conditionnelles telles qu'elles étaient accordées à cette époque. Cette recommandation fut de loin la plus controversée de son rapport et elle suscita une opposition 
farouche de toutes les instances qui étaient impliquées dans l'application des diverses mesures de libération conditionnelle. Ces instances étaient nombreuses et comprenaient, entre autres, la Commission nationale des libérations conditionnelles rattachée au ministère fédéral du Solliciteur général et les trois commissions provinciales des libérations conditionnelles. En outre, divers organismes privés, comme la Société JohnHoward, étaient impliqués dans l'application des programmes de libération conditionnelle. On comprendra sans peine que toutes les personnes composant ces organismes allaient résister de façon opiniâtre à l'abolition de leur chasse-gardée.

Il apparut rapidement que le gouvernement était sensible aux arguments déployés par les partisans de la libération conditionnelle. En effet, le ministère de la Justice chargea un ancien directeur de la Commission nationale des libérations conditionnelles d'organiser une consultation d'experts sur les propositions de la CCDP. Comme il fallait peut-être s'y attendre, cette consultation déboucha sur une réaffirmation de la nécessité des libérations conditionnelles.

Nous n'aimerions pas laisser l'impression que le débat entre partisans et adversaires des libérations conditionnelles peut être réduit à une simple épreuve de force où les premiers défendent leur territoire. Les avocats de la pratique des libérations conditionnelles disposaient d'arguments très sérieux qu'on ne saurait d'aucune façon réduire à une rhétorique bureaucratique défensive.

\subsection{Le désastre américain}

Depuis le début des années 1970, plusieurs États des États-Unis avaient aboli les libérations conditionnelles sous l'impulsion d'un mouvement de retour vers le rétributivisme. Dans sa version contemporaine, ce mouvement avait pris l'appellation de Just deserts («juste dû»), et il était caractérisé, entre autres aspects, par son scepticisme à l'égard de la possibilité d'utiliser l'incarcération pour réhabiliter les détenus. Ce scepticisme se traduisit par une critique impitoyable des sentences indéterminées, où c'étaient des commissions de libération conditionnelle qui déterminaient en pratique la durée des sentences d'incarcération. Par voie de conséquence, le retour vers les sentences déterminées s'accompagna aux États-Unis de propositions pour abolir la pratique des libérations conditionnelles. 
Dans sa conception initiale, le modèle du juste dû impliquait que l'abolition des libérations conditionnelles devait nécessairement être compensée par une diminution marquée de la longueur des sentences d'incarcération imposées par les juges. Or, dans beaucoup d'États américains, on se contenta d'abolir les libérations conditionnelles sans compenser cette mesure par une réforme du sentencing. Ce déséquilibre provoqua une augmentation effarante de la population carcérale aux États-Unis. D’après les statistiques les plus récentes, cette population est passée de 270 000, au début des années 1970 à 1,2 million aujourd'hui. L'abolition des libérations conditionnelles n'est évidemment pas le seul facteur qui rende compte de la croissance exponentielle de la population incarcérée aux États-Unis. La croisade déclenchée contre le trafic des stupéfiants par le président Bush a sûrement déterminé une augmentation de la population carcérale. Cependant, la proportion précise de cette augmentation n'a pas encore été déterminée, alors que l'on a réussi à calculer l'effet de l'abolition des libérations conditionnelles, qui est la cause la plus fréquemment citée par les chercheurs du problème de la surpopulation carcérale.

On oublia que les réformateurs qui s'inspiraient du rétributivisme (juste dû) avaient exigé que l'abolition des libérations conditionnelles déclenche une réforme à la baisse de la sévérité des sentences. En bons stratèges, les partisans des libérations conditionnelles affirmèrent que le mouvement du juste dû conduisait nécessairement à une croissance cancéreuse des populations incarcérées. Comme le rapport de la CCDP manifestait de la sympathie pour la version moderne du rétributivisme, on prétendit que l'application de ses recommandations conduirait inévitablement à une augmentation démesurée du nombre des personnes incarcérées. Cet argument se révéla un puissant soutien pour disqualifier auprès de certains les propositions de la CCDP.

Répétons en conclusion qu'on aurait tort de voir dans l'argument précité un pur artifice. Les craintes que l'abolition des libérations conditionnelles ne conduise à une progression exponentielle de la population carcérale étaient très certainement en partie fondées. Le modèle proposé par la CCDP demeure à notre avis viable. Il ne faudrait toutefois pas s'en dissimuler les risques. Toute mesure qui restreint l'accession des détenus à une libération anticipée doit être compensée par une réforme du sentencing qui procède à une désescalade des sanctions. L'instrument premier de cette réforme doit être la création d'une commission de sentencing qui élabore des lignes directrices à l'intention des juges. 


\subsection{Les relations fédérales-provinciales}

Les facteurs que nous avons jusqu'ici invoqués sont de nature à expliquer qu'aucune des propositions de la CCDP n'ait été jusqu'ici appliquée. Le gouvernement s'est jusqu'ici contenté de donner au rapport de la CCDP le suivi bureaucratique rituel. Nous allons maintenant élargir le débat en identifiant un facteur qui constitue à nos yeux un obstacle à toute réforme pénale au Canada.

Commençons par rappeler que le gouvernement fédéral n'a juridiction que sur les établissements carcéraux où des détenus servent des sentences de plus de deux ans. Les gouvernements provinciaux ont juridiction sur les prisons où les gens sont incarcérés pour une durée de moins de deux ans. La détermination de cette frontière de deux ans est le fruit adventice d'une conjoncture historique qui s'est développée au cours du XIX siècle (voir le chapitre II du rapport de la CCDP).

Cette répartition des compétences juridictionnelles a maintenant pris une signification qui dépasse de beaucoup la simple question de l'incarcération. Notons d'abord que le nombre des personnes incarcérées dans des établissements provinciaux est environ trois fois plus élevé que celui des personnes incarcérées dans des pénitenciers fédéraux. Les problèmes de surpopulation carcérale sont sans doute encore plus aigus dans les prisons provinciales que dans les établissements fédéraux. On doit en conclure que toute mesure susceptible d'accroître la pression démographique sur les établissements provinciaux intéresse au premier chef le gouvernement des provinces.

Depuis 1987, la question primordiale au niveau du sentencing a considérablement évolué. Tout ce que nous avons précédemment dit démontre que les propositions de la CCDP ont été parfaitement étrangères à cette évolution. Pour l'essentiel, le facteur déterminant dans cette évolution est l'ampleur très considérable que commencent à prendre les sanctions dites intermédiaires aux États-Unis et au Royaume-Uni. En effet, lors des travaux de la CCDP, les questions controversées concernaient avant tout l'abolition des libérations conditionnelles et les mesures substitutives à l'incarcération (les «alternatives à l'incarcération»). À l'heure présente, ce sont les sanctions dites intermédiaires, par exemple l'assignation à domicile sous surveillance électronique, qui constituent les mesures les plus chaudement discutées (voir Petersilia, 1987, et surtout Morris et Tonry, 1990). Les sanctions intermédiaires s'inscrivent, comme leur nom l'indique, entre la privation complète de liberté (incarcération) et la sentence qui n'implique aucune privation de 
la liberté au sens physique du terme (par exemple l'amende). Dans cette mesure, la sanction intermédiaire est différente de la peine substitutive à l'emprisonnement. Cette dernière est une mesure alternative à l'incarcération. Cependant, la sanction intermédiaire est moins une mesure qui se substitue à l'incarcération qu'une variante moins rigoureuse et moins coûteuse de la privation de liberté. Les sanctions intermédiaires partagent toutefois un trait commun extrêmement important avec les mesures substitutives à l'incarcération. Tant les sentences alternatives que les sanctions intermédiaires ne peuvent pas être imposées, à l'heure présente, à la place de sanctions lourdes d'incarcération. Pour le dire autrement, ces mesures ne peuvent «remplacer» que des peines d'incarcération de moins de deux ans. En conséquence, tout le champ des sanctions intermédiaires tombe en théorie sous la juridiction des provinces, comme le reconnait explicitement le document de consultation de 1990 distribué par le ministère de la Justice (voir le document portant sur la détermination de la peine, p. 22).

La conclusion ultime de la discussion qui précède est que les principaux enjeux de la réforme pénale concernent avant tout les provinces. Le partage des compétences entre le fédéral et le provincial s'effectue, nous l'avons vu, essentiellement en fonction de la durée des sentences d'incarcération. La conséquence ruineuse de cet accident de l'histoire canadienne est que le gouvernement fédéral n'est directement concerné que par les mesures d'incarcération et de remise en liberté anticipées. Cette conséquence se prolonge elle-même d'un puissant effet de renforcement de l'incarcération au Canada. En effet, la compétence fédérale en matière correctionnelle se définit entièrement par rapport aux peines d'incarcération les plus lourdes.

En d'autres termes, le gouvernement fédéral est «hors du coup» en ce qui concerne les principales préoccupations qui animent les réformateurs du système pénal. Ceux-ci accordent de plus en plus d'importance aux sanctions intermédiaires.

Or, le moins que l'on puisse dire à l'heure actuelle, c'est que les relations fédérales-provinciales ne baignent pas dans l'huile et qu'elles sont même empreintes d'une certaine humeur chafouine. Conclure que les enjeux de la réforme pénale concernent en droit d'abord les provinces équivaut à reconnaître qu'il sera très difficile de procéder à cette réforme. Il est d'une part douteux que les provinces disposent par elles-mêmes de la volonté politique d'entreprendre cette réforme. C'est plutôt le gouvernement fédéral qui a exercé, à tout le moins au niveau 
des propositions de réforme, un leadership. Or, le leadership du gouvernement fédéral est présentement remis en question dans tous les domaines.

Pour que la réforme pénale ait quelque chance d'advenir, il est impérieux que le gouvernement fédéral retrouve une compétence fondamentale dans tout le champ de la pénologie. Ce résultat ne pourra être atteint que lorsque l'on aura redéfini la ligne de partage entre les compétences correctionnelles fédérales et les compétences correctionnelles provinciales. Autrement dit, le gouvernement fédéral doit sortir $\mathrm{du}$ ghetto juridictionnel carcéral où l'histoire canadienne l'a circonvenu.

\subsection{La consultation infinie}

Nous empruntons ce dernier titre à un ouvrage célèbre de Maurice Blanchot intitulé L'entretien infini. Il semble en effet que les gouvernements tentent de pallier le présent blocage par l'institution de palabres dont on espère qu'elles auront un résultat magique. On semble en effet croire qu'en empilant rapports de consultation par-dessus rapports de commissions d'enquête, tout cet entassement de mots se métamorphosera en une montagne de réalités. Les travaux que nous avons nousmême conduits sur les commissions d'enquête ont montré que cette stratégie était effectivement poursuivie et qu'elle ne manquait pas d'efficacité (Brodeur, 1984). Les passions que soulèvent les matières pénales contrastent de façon singulière avec le manque de volonté politique et populaire pour résoudre les questions urgentes qu'elles soulèvent.

\section{La présente consultation conduite par le ministère de la Justice}

Comme nous l'avons déjà dit dans notre introduction, le ministère fédéral de la Justice a publié deux documents intitulés Vers une réforme. Le premier de ces documents porte sur La détermination de la peine et le deuxième sur Les affaires correctionnelles et la mise en liberté sous condition. Nous aimerions commenter brièvement ces deux documents dans la seconde partie de nos remarques. Nous commenterons à la suite les deux documents et nous leur accorderons une place sensiblement égale. Nous nous référerons à ces deux documents de consultation en usant des sigles DC-1, pour le document sur la détermination de la peine, et 
DC-2, pour celui qui porte sur les affaires correctionnelles et la mise en liberté sous condition.

\subsection{Le document sur la détermination de la peine}

Ce premier document comporte six propositions de réforme, que nous citerons textuellement:

a) l'incorporation dans la loi d'un Énoncé des objectifs et des principes de la détermination de la peine;

b) la création d'une commission permanente sur la détermination de la peine et les libérations conditionnelles;

c) l'adoption d'un code de preuve et de procédure aux fins de l'audience relative à la détermination de la peine;

d) l'élaboration d'un nouveau mode d'infliction et de perception des amendes;

e) la réorganisation de la Partie XXIII du Code criminel;

f) l'adoption de sanctions intermédiaires. (DC-1: 4)

Nous commenterons les recommandations $a, b$, $d$ et $f$. Les recommandations $\mathrm{c}$ et e concernent des aspects plus formels de la détermination de la peine qui sont d'un intérêt plus immédiat pour les juristes. L'effort de clarification impliqué par ces recommandations nous apparait toutefois nécessaire.

Les questions qui sont posées dans le document de consultation sont pertinentes et, pour la plupart, elles sont également judicieuses. Elles sont toutefois répétitives et soulèvent à nouveau des points qu'on pouvait espérer avoir réglés. En outre, le document de consultation même est dicté par une volonté de conciliation. Or, il n'est pas sûr que cette volonté de conciliation produise autre chose qu'un résultat relativement superficiel selon lequel les contradictions qui sont résolues sur papier reviendront avec insistance dans la pratique.

\subsubsection{L'énoncé des objectifs et des principes}

Les remarques que nous venons de faire s'appliquent de façon particulière à l'énoncé des objectifs et des principes.

En effet, cet énoncé reprend des positions énoncées en 1982 dans un document intitulé Le droit pénal dans la société canadienne et en 1984 dans le Livre blanc sur la détermination de la peine. Ces deux documents avaient donné lieu à une première formulation d'un Énoncé des 
objectifs et des principes de la détermination de la peine qui fut soumis au Parlement dans le projet de loi C-19. Bien que ce projet mourût au feuilleton de la Chambre, il n'en fut pas moins largement discuté au Canada. Il sembla se dégager à l'époque un consensus pour qu'un ordre de priorité fût déterminé parmi les principes formulés dans l'Énoncé (le projet de loi C-19 fut précisément critiqué parce qu'il ne déterminait pas un tel ordre de priorité). Or, l'on constate que le document de consultation (DC-1: 9) hésite encore à hiérarchiser ces principes et qu'il sollicite des avis sur l'opportunité d'établir un ordre de priorité. On pensait cette question résolue.

Le modèle adopté dans DC-1 pour la formulation des objectifs de la détermination de la peine constitue un retour en arrière vers les propositions du projet de loi C-19, cautionnées à nouveau en 1988 par le CJSG présidé par monsieur David Daubney. Ce modèle tient dans l'énoncé d'un objectif fondamental - la protection de la société - qui peut être diversement atteint par l'adoption de toute une série de sousobjectifs. Ces objectifs subordonnés ne sont rien d'autre que la liste des fins traditionnelles de la détermination de la peine - la dénonciation, la prévention générale, la prévention spéciale, la neutralisation et la réadaptation - agrémentée d'un nouvel objectif de réparation. Le problème, mille fois dénoncé, que soulève cette liste ouverte est qu'elle autorise tout, même et surtout des pratiques contradictoires, et qu'elle institutionnalise en droit l'exercice complètement débridé de la discrétion des magistrats.

\subsubsection{La commission sur la détermination de la peine et les libérations conditionnelles}

Si l'on décide de préserver les libérations conditionnelles dans leur forme actuelle, il est souhaitable que le sentencing et les pratiques de libération anticipée fassent l'objet d'une approche concertée. Le document de consultation déclare à cet égard qu'«il faut absolument que les questions de la détermination de la peine et de la libération conditionnelle soient traitées conjointement si l'on veut que le système atteigne les objectifs de l'intelligibilité et de l'accessibilité» (DC-1: 10).

Rien n'assure toutefois que la nouvelle commission puisse orchestrer cette concertation. Mentionnons d'abord que cette commission serait constituée de deux comités distincts dont les mandats porteraient respectivement sur la détermination de la peine et sur les libérations 
conditionnelles. Cette subdivision ne serait pas nécessairement préjudiciable à la concertation, mais elle pourrait le devenir.

Il y a toutefois plus grave. Les deux premiers objectifs de la commission sont formulés comme suit:

a) mettre au point les lignes directrices proposées par la Commission canadienne sur la détermination de la peine;

b) fournir des conseils en ce qui a trait à l'orientation générale des politiques, des critères et des lignes directrices applicables aux libérations conditionnelles. (DC-1: 12)

Il importe de voir que ces deux premiers objectifs sont profondément asymétriques. En effet, l'objectif premier de la composante sentencielle de la Commission est de mettre elle-même au point (le texte anglais dit develop) les lignes directrices proposées par la CCDP en 1987. Or, ces lignes directrices ne tiennent pas dans des principes abstraits et relativement vagues: elles définissent des présomptions relatives à la nature des mesures à imposer (incarcérer ou pas) et au quantum de ces mesures (la durée de l'incarcération).

Par contre, le mandat de la composante de la commission qui s'occupera des libérations conditionnelles est beaucoup moins précisément défini : cette composante n'élabore pas par elle-même des lignes directrices sur les libérations conditionnelles. Elle se contente de «fournir des conseils en ce qui a trait à l'orientation générale» de politiques, critères et lignes directrices applicables aux libérations conditionnelles (le texte anglais utilise l'expression to advise au lieu de to develop). En d'autres termes, la commission élabore des politiques en ce qui a trait à la détermination des peines, alors qu'elle est confinée dans un rôle de conseiller s'agissant des libérations conditionnelles.

Cette interprétation n'est pas la seule possible. Le texte du document propose le commentaire suivant sur la nature des lignes directrices en matière de sentencing: "Ces lignes directrices traiteraient des écarts injustifiés eu égard à la race et au sexe des contrevenants.» (DC-1:11) Si tel est le contenu des lignes directrices en matière de détermination des peines, elles se rapprochent des orientations générales qui seront proposées par la commission aux organismes qui gèrent les libérations conditionnelles. Ce progrès dans la symétrie n'est toutefois acquis qu'au profit d'une liquidation de la conception des lignes directrices en matière de sentencing que la CCDP a élaborée dans son rapport de 1987. 
De deux choses l'une. Ou bien le mandat de la nouvelle commission présente un sérieux déséquilibre, auquel cas il est douteux qu'elle puisse travailler de façon concertée, ou bien son mandat est homogène et relativement similaire pour les deux comités qui la composent, auquel cas la notion de lignes directrices en matière de détermination de la peine est vide de contenu.

\subsubsection{Les réformes en matière d'infliction et de perception des amendes}

Pour la plupart d'entre elles, ces réformes constitueraient un progrès significatif sur la situation actuelle, où l'on abuse de l'incarcération pour défaut de paiement d'amende. Ces réformes s'inspirent largement des propositions de la CCDP.

Ces réformes constituent néanmoins une régression sur un point important. Le rapport de la CCDP présentait une table d'équivalences entre le montant des amendes et les peines d'incarcération qui devaient être purgées dans le cas d'un défaut de paiement d'amende (section 12.26 du rapport de la CCDP). L'établissement de cette table d'équivalences reposait sur le présupposé que le contrevenant incarcéré pour défaut de paiement d'amende liquidait le montant de sa dette monétaire envers l'État en purgeant sa sentence d'incarcération.

Or, le document de consultation adopte une position contraire en cette matière:

En outre, tenant pour acquis que l'emprisonnement en pareilles circonstances est une mesure d'exécution et non une autre forme de peine, nous proposons que l'incarcération du contrevenant ne remplace pas l'amende ordonnée initialement et que le contrevenant en question soit toujours tenu de payer l'amende même après avoir été incarcéré. (DC-2: 16; c'est nous qui soulignons)

Nous estimons que cette recommandation est rétrograde et qu'elle rappelle fâcheusement la fameuse disposition sur le remanet selon laquelle un contrevenant ne se voyait pas reconnaître le temps qu'il avait passé en liberté surveillée si sa libération conditionnelle était révoquée avec l'expiration de sa sentence (le temps passé en liberté surveillée comptait pour rien). Une sentence ne doit jamais fournir l'amorce d'un processus d'escalade pénale, qui superpose les peines. 


\subsubsection{Les sanctions intermédiaires}

Cette partie du document de consultation constitue une innovation par rapport aux travaux des commissions précédentes et ses propositions sont intéressantes. Elles se butent toutefois à l'obstacle des juridictions partagées entre le gouvernement fédéral et les provinces que nous avons décrit précédemment. Au lieu de répéter ce que nous avons déjà dit à ce sujet, nous citerons un passage du document de consultation qui confirme nos appréhensions:

Le gouvernement fédéral ne peut pas prendre de mesures définitives dans le domaine des sanctions intermédiaires. L'élaboration d'une série de mesures qui serviront à la fois les intérêts des provinces et ceux du gouvernement fédéral est d'une importance capitale. Nous ne pouvons imposer aux provinces des exigences qui auront pour effet d'entraîner des dépenses considérables sur le plan des programmes sans mener des consultations exhaustives. D'un autre côté, nous ne pouvons prendre des engagements qui obligeraient le gouvernement fédéral à consacrer des sommes importantes à ces programmes et maintenir en même temps notre position sur le plan fiscal. Nous devons mener des consultations exhaustives auprès des administrations concernées afin de trouver des solutions qui conviennent d'une part et d'autre. (DC-2: 22; c'est nous qui soulignons)

Ce passage appelle trois remarques:

a) Il semble bien que la difficulté juridictionnelle que nous avons identifiée par rapport aux sanctions intermédiaires soit réelle.

b) L'objectif premier des sanctions intermédiaires est de punir à un moindre coût que l'incarcération. On doit s'étonner que ce passage ne discute que des coûts nouveaux que susciteront les sanctions intermédiaires sans même mentionner les économies qu'elles provoqueront. Se pourrait-il que ces sanctions soient moins économiques qu'on ne l'a d'abord cru? Les expériences menées en ColombieBritannique avec l'assignation à domicile sous surveillance électronique semblent suggérer que oui.

c) La répétition de l'expression "consultations exhaustives» nous semble témoigner de la pensée magique dont nous avons décrit l'opération plus haut. Le mot "exhaustif» est peut être associé à l'aspect «compréhensif» de ces négociations éventuelles. Il peut également signifier leur caractère épuisant, dont on pensa jadis qu'il suscitait des compromis heureux. 


\subsection{Le document sur les affaires correctionnelles et la mise en liberté sous condition}

De façon relativement inattendue, ce second document (DC-2) est beaucoup plus détaillé dans ses propositions que le premier. Le processus de réforme ayant eu pour objet premier la détermination des peines, on se serait attendu que le document portant sur cette partie de la réforme soit plus explicite que le second. Il n'en est pas ainsi. L'une des raisons en est que les réformes en matière correctionnelle sont moins tributaires de changements législatifs que les premières et qu'on peut y procéder sur le plan administratif. C'est pourquoi le second document ne se borne pas seulement à présenter des réformes futures. Il décrit un processus de changement qui fonctionne depuis plus de cinq ans.

Un nombre important de ces changements constitue d'importants progrès, comme la clarification des missions des divers services correctionnels. L'espace nous manque pour rendre pleine justice à cet intéressant document. Nous nous bornerons à trois remarques, où l'on reconnaîtra des préoccupations qui nous sont propres.

\subsubsection{Incarcération et réinsertion sociale}

Les deux documents de consultation ne sont pas pleinement convergents en ce qui concerne les vertus réformatrices de l'incarcération. Le document de consultation sur la détermination de la peine contient le passage suivant:

L'emprisonnement coûte cher et il ne sert pas à grand-chose, si ce n'est à garder les contrevenants à l'écart de la société pendant un certain temps. (DC-1: 19)

Le second document sur les affaires correctionnelles comporte un Énoncé de l'objet et des principes de l'administration des services correctionnels fédéraux. Une partie de cet énoncé affirme que l'administration correctionnelle a pour objet de protéger le public,

[...] en contribuant à la réadaptation des délinquants dans la collectivité à titre de citoyens respectueux de la loi, en leur fournissant des programmes dans les pénitenciers et dans la collectivité. (DC-2: 32 ; c'est nous qui soulignons)

Bien que nous comprenions qu'il est peut-être impossible pour un service de reconnaître qu'il ne poursuit pas le bien de sa clientèle, nous pensons que cette compulsion à réaffirmer l'existence de programmes 
qui favorisent la réadaptation des détenus incarcérés dans les pénitenciers ne fait que perpétuer une illusion nocive à tout effort soutenu pour faire reculer l'incarcération. Tout se passe comme si l'incarcération était considérée comme un mal nécessaire à l'étape de la sentence et comme un mal suspendu après l'emprisonnement du contrevenant.

\subsubsection{La croisade contre la drogue}

Le document prévoit que certains contrevenants devront purger l'entièreté de leur sentence d'emprisonnement. Actuellement, seuls les contrevenants qui présentent un risque élevé de commettre un délit violent une fois remis en liberté peuvent être bloqués en prison jusqu'à l'expiration complète de leur sentence.

Le document de consultation propose d'ajouter à la catégorie des contrevenants susceptibles d'être bloqués ceux qui ont été reconnus coupables d'un délit grave lié à la drogue et dont la sentence, telle que déterminée par le juge, prévoit explicitement qu'ils ne seront pas admissibles à une libération conditionnelle avant d'avoir purgé la moitié de leur peine d'emprisonnement.

L'une des causes de l'accroissement démesuré de la population carcérale aux États-Unis est la douteuse croisade entreprise contre la drogue (à l'exclusion de l'alcool et de la nicotine). La tendance canadienne à ne retenir que ce qu'il y a de pire dans l'expérience pénale américaine est difficile à expliquer. Elle est tout à fait fâcheuse.

\subsubsection{Libération conditionnelle et protection de la société}

Le rapport de la CCDP soutient, selon nous avec raison, que la protection de la société est fondamentalement un moyen de légitimer l'incarcération. Il devrait s'ensuivre, si cet aperçu est juste, qu'il est jusqu'à un certain point contradictoire de prétendre que l'objectif des libérations conditionnelles réside dans la protection de la société. L'interprétation la plus immédiate et la plus répandue de l'impératif de protéger la société conduit à la ségrégation des contrevenants, et non à leur remise en liberté.

Dans son Énoncé de mission et de valeurs de 1986, la Commission nationale des libérations conditionnelles a tenté de justifier la remise en liberté anticipée par la contribution de cette mesure à la protection de la société. Selon cet énoncé, la Commission nationale des libérations conditionnelles: 
[...] contribue à la protection de la société en facilitant la réintégration opportune des délinquants comme citoyens respectueux de la loi. (Cité dans DC-2: 6)

Dans un article récent (Brodeur, 1990), nous avons tenté de montrer que cette position était intenable et que seule la pratique systématique de l'ambiguité permettait de s'y retrancher. Les recommandations du second document de consultation (DC-2) lèvent définitivement le paradoxe de fonder une pratique de remise en liberté sur un objectif qui pactise essentiellement avec l'incarcération. Ce document présente un Énoncé de l'objet et des principes généraux de la libération conditionnelle au Canada. Il commence d'abord par proposer que la protection du public soit le principal facteur à considérer au moment de décider de remettre un prisonnier en liberté avant l'expiration de sa sentence d'incarcération. Puis, dans le droit fil de la logique de la protection du public, le document reprend les critères sur lesquels devrait être fondée la décision de remettre un prisonnier en liberté (ces critères furent initialement proposés par les quatre commissions de libérations conditionnelles dans le cadre de la réforme du droit correctionnel):

La mise en liberté du détenu:

a) ne représente pas un risque trop grand, c'est-à-dire que le détenu ne récidivera pas, avant l'expiration de sa peine actuelle telle que prévue par la loi, en commettant un acte criminel risquant de gravement porter atteinte au public;

b) contribuera à la protection de la société en favorisant la réintégration du détenu en tant que citoyen respectueux de la loi ou en rendant possible la continuation de cette réintégration, ce qui aura du même coup pour effet de satisfaire aux objectifs du système de justice. (DC-2: 9)

Pour lever tout malentendu par rapport à l'ordre de priorité selon lequel ces critères doivent être appliqués, le document apporte la précision suivante, dont l'importance est cruciale:

Ces critères sont conformes à l'énoncé proposé de l'objet et des principes et à l'esprit des recommandations formulées par le Comité permanent [note: il s'agit du CJSG, présidé par D. Daubney]. Le gouvernement considère comme essentielle l'inclusion, dans toute réforme de la mise en liberté sous condition, de critères fondés spécifiquement sur le risque. S' ils sont acceptés, ces critères seront appliqués par l'ensemble des commissions des libérations conditionnelles au Canada. (DC-2: 9 ; c'est nous qui soulignons) 
Le document de consultation poursuit en recommandant de préciser le texte législatif de manière à manifester sans équivoque que la mission des commissions des libérations conditionnelles consiste dans l'évaluation des risques que présente la libération anticipée du détenu:

Pour chacun de ces programmes [de remise en liberté anticipée], l'accent serait mis sur le risque de récidive après la mise en liberté. (DC-2: 10)

En termes clairs, la logique de la protection de la société et du public fournit une structure de légitimation du refus plutôt que de l'acceptation de la remise en liberté.

Il importe de rappeler le sens de nos remarques. Elles ne sont aucunement dirigées contre la libération conditionnelle mais contre l'inconséquence qu'il y a à justifier cette pratique dans le cadre d'une logique qui l'empêche plutôt qu'elle ne la favorise.

\section{Conclusion}

Ce texte s'est déjà trop allongé et nous conclurons en quelques phrases. Le principal reproche que nous faisons aux propositions contenues dans les deux documents de consultations de 1990 est de dissoudre le contenu des réformes envisagées en tentant de les rendre acceptables à toutes les parties. Il faudrait user de principes de rigueur et de parcimonie dans la volonté de réformer le système pénal en prenant garde de ne pas multiplier les tentatives qui sont manifestement d'effets pervers. Pour insatisfaisant qu'il soit, le statu quo est préférable à une réforme cosmétique, car il constitue un rappel incessant de la nécessité de le faire éclater en posant des choix clairs.

\section{Références}

Brodeur, J.-P. (1984). La délinquance de l'ordre. Montréal: Hurtubise HMH.

Brodeur, J.-P. (1990). The attrition of Parole. Canadian Journal of Criminology, vol. 32 (3), 503-510.

Canada (1990). Vers une réforme: La détermination de la peine. Ministère de la Justice, Ottawa, Ministère des Approvisionnements et Services Canada. (Cité sous le sigle DC-1)

Canada (1990). Vers une réforme: Les affaires correctionnelles et la mise en liberté sous condition. Ministère de la Justice, Ottawa, Ministère des Approvisionnements et Services Canada. (Cité sous le sigle DC-2) 
Canada, chambre des communes (1988). Des responsabilités à assumer: Rapport du Comité permanent de la justice et du Solliciteur général sur la détermination de la peine, la mise en liberté sous condition et d'autres aspects du système correctionnel. Ottawa, Imprimeur de la Reine. (Cité sous le sigle CJSG)

Commission canadienne sur la détermination de la peine (1987). Réformer la sentence: Une approche canadienne, Ottawa, Ministre des Approvisionnements et Services Canada. (Cité sous le sigle CCDP)

Commission de réforme du droit du Canada (1987). Pour une nouvelle codification du droit pénal, rapport $\mathrm{n}^{\circ} 31$, édition révisée et augmentée, Ottawa.

Commission de réforme du droit du Canada (1989). Les discussions et ententes sur le plaidoyer, document de travail 60, Ottawa.

Morris, N., \& Tonry, M. (1990). Between Prison and Probation. New York: Oxford University Press.

Petersilia, J. (1987). Expanding Options for Criminal Sentencing. Santa Monica: Rand Corporation.

ABSTRACT - This review of recent developments in the field of sentencing in Canada begins by observing that none of the recommendations of the Canadian Sentencing Commission were implemented, since the commission issued its report in 1987. This amounts to a prolongation of the statu quo. We propose elements of explanation as to why there was no follow up to the Commission's proposais. Second, we present a critical analysis of the latest consultation package on sentencing and parole, that was put together by the federal Department of Justice in 1990. We argue that the proposed statement of purposes and principles of sentencing should priorize the different sentencing goals that it enumerates. We also point out that there is an unhalance between the sentencing and parole components of the permanent commission proposed by the Department of Justice. The sentencing component is required to make sentencing policy, whereas the parole component acts as an advisor to the National Parole Board.

KEYWORDS - Canadian Sentencing Commission, sentencing, sentencing policy, parole, National Parole Board, Department of Justice.

RESUMEN - Esta recensión de acontecimientos recientes en el campo de la determinación de la pena permite constatar que ninguna de las recomendaciones de la Comisión canadiense sobre la determinación de la pena se ha cumplido desde que dicha comisión publicó su informe de 1987. Ello ha implicado la prolongación del statu quo. Proponemos algunos elementos que explican por qué no hubo seguimiento a las propuestas de la Comisión. Segundo, presentamos un análisis crítico del informe de consultas sobre determinación de la pena y liberación condicional elaborado por el ministerio de Justicia federal en 1990. Argumentamos que la propuesta de declaración de objetivos y principios de la determinación de la pena debe dar prioridad a las diferentes metas sobre sentencias que ahí enumera. Destacamos también que existe un desequilibrio entre los componentes de la determinación de la pena y de la liberación condicional de la Comisión permanente propuesta por el ministerio de Justicia. El componente de determinación de la pena debe constituir una política de determinación de la pena, mientras que el componente de liberación condicional debe cumplir funciones de asesoría ante la Comisión nacional de liberaciones condicionales. 
PALABRAS CLAVE • Comisión canadiense sobre la determinación de la pena, política de determinación de la pena, liberación condicional, Comisión nacional de liberaciones condicionales, ministerio de Justicia de Canadá. 